\title{
Performance tests to modeling future climate-vegetation interactions in virtual world: an option for application of remote sensed and statistical systems
}

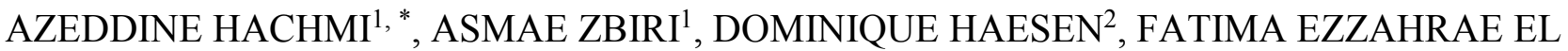 \\ ALAOUI-FARIS $^{1}$, DAVID A.VACCARI ${ }^{3}$
}

\author{
${ }^{1}$ Department of Biology \\ Mohammed V University, Faculty of Science \\ MOROCCO \\ ${ }^{2}$ Vlaamse Instelling Voor Technologisch Onderzoek (VITO) \\ BELGIUM \\ ${ }^{3}$ Stevens Institute of Technology, Hoboken, NJ, Civil, \\ Environmental and Ocean Engineering \\ UNITED STATES
}

\begin{abstract}
Working in the virtual world is different to real experiment in field. Nowadays, with remote sensing and new analysis programs we can assure a quick response and with less costs. The problem is efficiency of these methods and formulation of an exact response with low errors to manage an environmental risk. The objective of this article is to ask question about performance of some tools in this decision making in Morocco. The study uses (Test 1: TaylorFit Multivariate Polynomial Regressions (MPR); Test 2: SAS Neural Network (NN) to modeling relationship between European Center for Medium-Range Weather Forecasts dataset and NDVI eMODIS-TERRA at arid Eastern Morocco.

The results revealed that the both test could accurately predict future scenario of water stress and livstock production decrease. The experience shows that virtual work with Artificial Intelligence is the future of ecological modeling and rapid decision-making in case of natural disasters.
\end{abstract}

Key-Words: - Performance Tests, Decision making, Virtual world, Multivariate Polynomial Regressions (MPR); Neural Network (NN), NDVI, ECMWF Rainfall, Eastern Morocco.

Received: March 2, 2021. Revised: December 3, 2021. Accepted: December 21, 2021. Published: December 31, 2021.

\section{Introduction}

\subsection{Understand problems}

Natural disaster (flood, drought, forest fire) are phenomenons related to climate change, exacerbated by human activities, for months and years. Globally and in Morocco, food security in arid and semi-arid areas is/will be faced, due to climate change. The challenges are decreasing water resources, grazing potential and increasing needs of these populations. The water stress map shows that Morocco is affected by between $25 \%$ and $70 \%$ water stress. In Morocco meteorological trends show a decrease in precipitation and an increase in temperatures (Gommes et al., 2009; Hofste et al., 2019) [1, 2].

This water-related phenomenon affects a wide range of environmental and vegetation factors (Dutta et al., 2015) [3]. Overgrazing impacts floristic composition and it also causes a decrease in perennial plant cover and development of invasive plant species (Hachmi et al., 2018) [4]. This precarious situation worsens during periods of severe drought when a significant water deficit is observed. Global demand for livestock products is expected to double by 2050, mainly due to improving global living standards. Meanwhile, climate change is a threat to forage production, water availability, animal reproduction, and biodiversity (Rojas-Downing et al., 2017) [5].

On an area of 290,000 ha, the study area includes the routes of Bni Mathar, Mrija, Gafait and Tiouli. The natural environment is characterized by a fragility and vulnerability, requiring urgent conservation and rehabilitation measures. Indeed, the ecological potential of this area is strongly determined by the aridity. The bioclimatic environment arid medium to mild winter is dominant (Hachmi et al., 2018) [4]. The soils in the study area are silty to clayey, generally not very developed, shallow and poor in organic matter. Rainfall is low and irregular, varying from less than 50 to $350 \mathrm{~mm}$. The average annual rainfall is 200 $\mathrm{mm} /$ year over the entire area. Average annual 
temperatures in the region are around $19^{\circ} \mathrm{C}$ with a minimum of $17.5^{\circ} \mathrm{C}$ and a maximum of $23^{\circ} \mathrm{C}$ (Berkat et al., 2004, Zbiri et al., 2021) [6, 7]. The main plant formations of this area are grassy steppes dominated by Macrochloa tenacissima and chamaephytic steppes dominated by Artemisia herba alba. We find also, chenopodiaceous steppes and halophyte facies (Salsola sp, Atriplex halimus). Chronic overgrazing has led to the almost total elimination of palatable species of Artemisia herba alba and Macrochloa tenacissima and the installation of aggressive species with little or no palatability or even toxicity (Anabasis aphylla, Noaea mucronata, Atractylis serratuloides, Peganum harmala, Lygeum spartum) (Hachmi et al., 2018; Zbiri et al., 2021) [4, 7].

\subsection{Spatio-temporal Overview}

Recently, remote sensing data have been able to systematically and continuously provide information such as vegetation and water cycles (Xu et al., 2018). For several years, land cover change detection using remote sensing data largely focused on capturing abrupt changes between two time intervals, where one land cover type is replaced by another (Coppin et al., 2004). Over time, however, improvements to instruments and data availability and advancements in algorithms and computation methods allowed for the development of different change-detecting techniques, such as time series analysis and temporal trajectory-based change detection (Hecheltjen et al., 2014) [10]. As a result, change detection and land cover classification are no longer implemented as separate and independent activities as was typical in past studies, allowing for the extraction of more thorough and meaningful change-related information. Additionally, recent advances in cloud computing platforms, such as Google Earth Engine (GEE) (Gorelick et al., 2017) [11], Amazon Web Services (Jackson et al., 2010) [12], NASA Earth Exchange (Nemani, 2012) [13], which host petabyte-scale data archive and offer high performance cloud computing capability, make implementation of state-of-the-art algorithms over large areas feasible (Tamiminia et al., 2020) [14].

The eMODIS-TERRA sensor provides a spatial resolution that is both broad enough to study the entire region and fine enough to work at the regional and municipal scales. The reflectance ratio calculated from the satellite remote sensing data is used to calculate the density of vegetation indices according to the following equation (Rouse et al., 1974) [15]:

\section{NDVI $=(\mathrm{NIR}-\mathrm{RED}) /(\mathrm{NIR}+\mathrm{RED})$ Éq 1.}

Where, NIR and RED are respectively the digital number value (ND) of near infrared and red.

Other studies on time series analysis of eMODIS NDVI data at low spatial resolution $(250 \mathrm{~m})$ have examined phenomena related to vegetation change at larger scales (Guo, 2002) [16]. Zbiri et al. (2019a) has tested whether the Moderate Resolution Imaging Spectroradiometer (MODIS) and emodis NDVI can be used to water stress in arid Moroccan rangelands [17]. Spatial remote sensing data are becoming instrumental in climate change detection, assessment and management. The analysis of the relationship between rainfall and NDVI is done (Nicholson \& Farrar, 1994 and Farrar et al., 1994; Thenkabail et al., 2004, Arshad et al., 2008, Hasan $\&$ Saiful, 2011, Brian et al., 2012) [18, 19, 20, 21, $22,23]$. These studies note significant differences in the relationship between NDVI and rainfall for different soil types. Martiny et al. 2006 demonstrated the sensitivity of NDVI to seasonal and interannual variations in rainfall in semi-arid région in Africa [24]. Rainfall estimates derived from satellite imagery and global circulation models are frequently used for vegetation monitoring in many areas of Africa because of the shortage of observed rainfall data and the sparse network of meteorological stations. At the same time, this scarce density of rain gauge stations makes the calibration and validation of the modelled data nearly impossible (Rojas et al., 2011) [25]. However, much work remains to be done to demonstrate how well this relationship will hold up in the face of climate change.

The European Centre for Medium-Range Weather Forecasting (ECMWF) is one of the best providers of high quality climate data series at different time scales (Woods, 2006) [26]. The interest in these data is justified by the fact that the FOODSEC action of the Joint Research Centre (JRC) is using them in an operational way for monitoring agricultural and pastoral vegetation on a real-time basis for the Horn of Africa (Rojas et al., 2005) [27]. In some cases it is also possible to use the rainfall and satellite data as NDVI for producing quantitative yield forecasts (Rojas, 2007) [28].

The year 2021 was among the seven warmest on record, and Europe experienced a summer of extremes, according to findings reported by the EU's Copernicus Climate Change Service implemented by ECMWF. Now for advancing global Numerical weather prediction (NWP) ECMWF have just added more than 50 new 
products to \#OpenCharts catalogue. These forecast charts are free for anyone to access, redistribute and adapt - even for commercial applications - part of their open data Strategy for 2021-2030 (https://www.ecmwf.int/) [29].

Numerical weather prediction uses mathematical models of the atmosphere and oceans to predict the weather based on current weather conditions.

\subsection{Statistical Artificial Intelligence}

\subsubsection{TaylorFit Response Surface Analysis (RSA)}

TaylorFit is a regression program that makes it easy to generate relatively simple equations to describe complex data. By "complex," we mean data that have nonlinear relationships, including interactions among multiple variables. This even extends to describing processes that have the behavior known as "chaotic."

The TaylorFit application works entirely client-side in your browser, so there's no need to download or install anything and no need to create an account. You can run the application simply by going to www.TaylorFit-RSA.com. Examples of situations that can be modeled with TaylorFit can be found everywhere - in business, engineering, and the sciences. One simple way to think of its applicability is to envision any situation with numerical information that can be collected into a table or spreadsheet with two or more columns of data, and with one column expected to depend upon the values in the other columns. Each row can be considered a distinct set of measurements repeated to form the set of rows (Vaccari, 2021) [32].

\subsubsection{SAS Predictive and Specialized Modeling}

Predictive and Specialized Modeling provides details about more technical modeling techniques, such as Response Screening, Partitioning, and Neural Networks.

The objective of this paper is to establish an accurate model of Multivariate Polynomial Regression and Neural Network using series of rainfall and NDVI images in the study area in eastern Morocco. Thus, to predict future hydrological and natural disaster scenarios with disaggregated rainfall anomaly between September and February.

\section{Data and Methodology}

\subsection{ECMWF data}

The rainfall data from 2001 to 2013 used in this work were taken from the European Centre for Medium-Range Weather Forecasts (ECMWF) with a spatial resolution grid of $11 \mathrm{~km}$ and a WGS-84 projection (Woods, 2006) [26]. These data are rescaled to a 0.25 degree grid and aggregated for periods of 10 days (decades). These images in ASCII format are georeferenced at the scale of the entire African continent with a resolution of approximately $10000 \mathrm{~m}$ (Fig. 2).

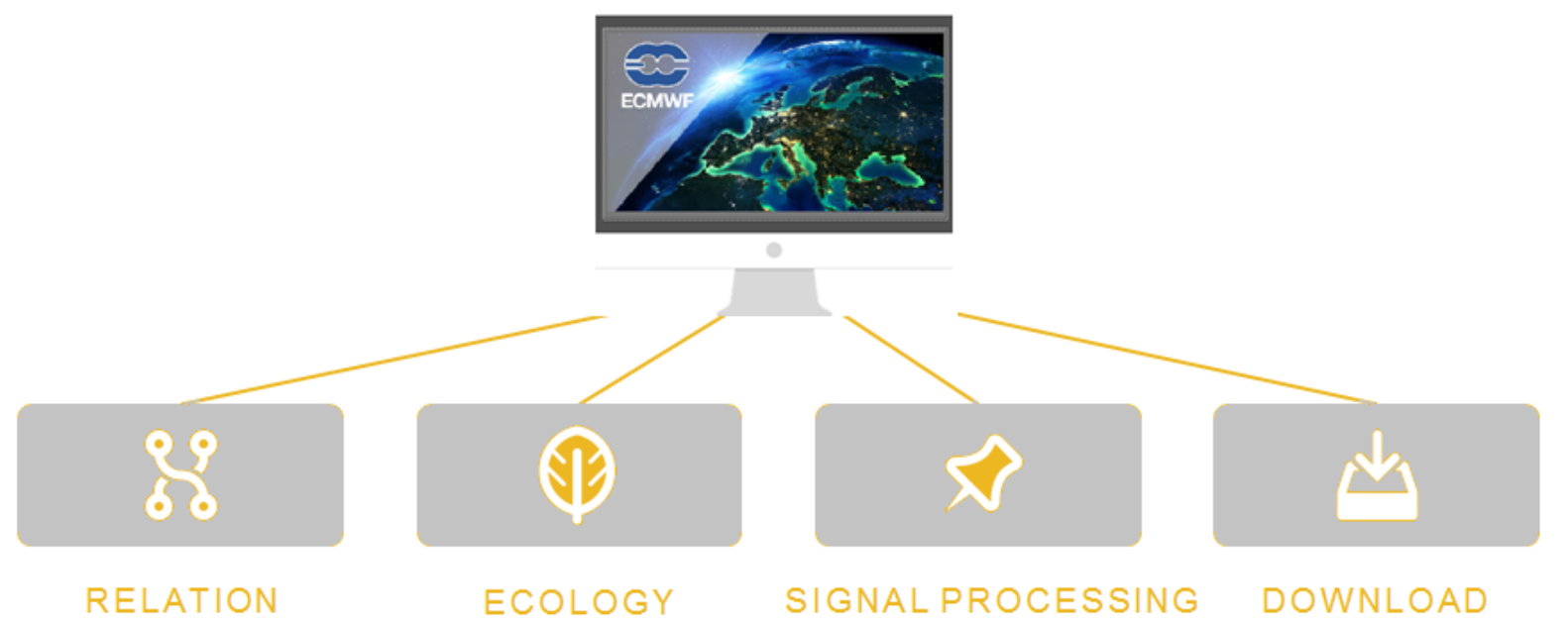

Fig 1. Steps to understand problems and try to solve them. 


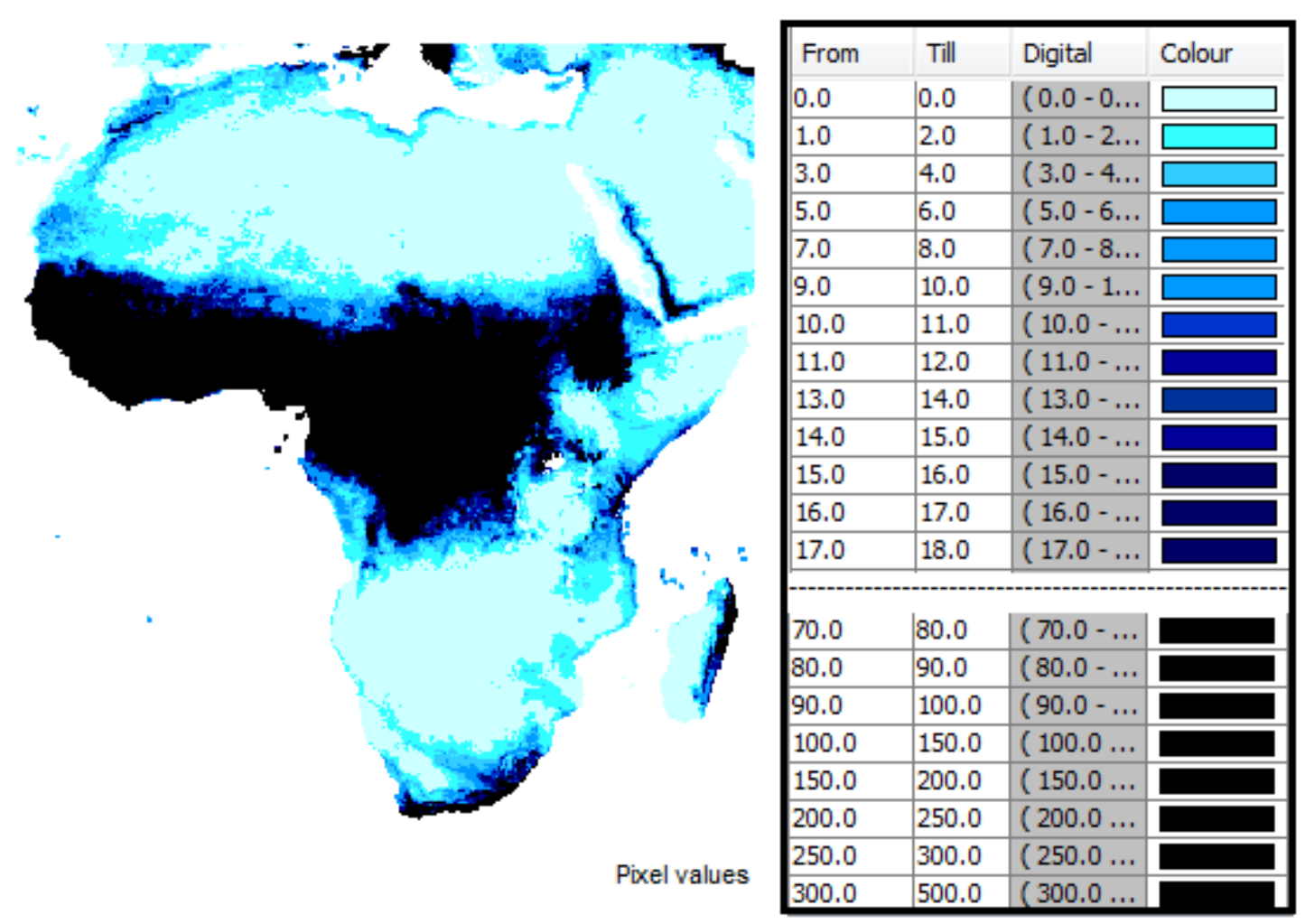

Fig 2. ECMWF rainfall estimate based on daily forecasts from ECMWF OPERATIONAL model (scaled from original resolution to 0.25 degrees by MeteoConsult).

\section{2 eMODIS data}

NDVI-eMODIS images with a spatial resolution of $250 \mathrm{~m}$ and a projection (WGS 84) were used. This time series of 552 NDVI eMODIS images dates from September 2001 to August 2013. The vegetation index is calculated from MODIS L1B Terra surface reflectances (Jenkerson et al., 2010) [30].

The NDVI and rainfall values of the European center are extracted per pixel with a Global Land Cover 2000 (GLC 2000) land cover mask. The extraction of the digital data from the satellite images was performed by SPIRITS (Software for the Processing and Interpretation of Remotely sensed Image Time Series) (Mayaux et al., 2004) [31].

\subsection{Multivariate Polynomial Regression (MPR)}

The type of models generates by TaylorFit are called Multivariate Polynomial Regression (MPR) Models. Consider the example above of agricultural crop yield (Y), which depends upon the amount of irrigation water (W) and fertilizer application rate (F). One of the best-known approaches for this problem is Multilinear Regression (MLR). TaylorFit is an extension of MLR to include interactions and other nonlinearities. The MLR model for this case would be:

$$
\mathrm{Y}=\mathrm{a}+\mathrm{b} \cdot \mathrm{W}+\mathrm{c} \cdot \mathrm{F} \text { Éq } 2 .
$$

This model has coefficients $a, b$, and $c$. The value of $\mathrm{b}$ indicates how much $\mathrm{Y}$ increases as $\mathrm{W}$ is increased. However, what if the effect of irrigation on yield depends upon how much fertilizer has been applied? This is an example of an interaction, and an MLR model cannot describe it.

However, if we add a term $\mathrm{d} \cdot \mathrm{W} \cdot \mathrm{F}$, the resulting model is capable of describing interactions:

$\mathrm{Y}=\mathrm{a}+\mathrm{b} \cdot \mathrm{W}+\mathrm{c} \cdot \mathrm{F}+\mathrm{d} \cdot \mathrm{W} \cdot \mathrm{F}$ Éq 3 .

A further problem may be that yield increases with $\mathrm{W}$ and with $\mathrm{F}$, but only up to a point. Then it saturates, or levels out. Plus, how fast yield saturates with irrigation may interact with fertilizer. The following model describes such a situation.

As described above, MLR is a widely understood methodology. Since TaylorFit response surface analysis (RSA) with stepwise multivariate polynomial regression (MPR) is a natural extension of MLR, it is a good fit for researchers who have used MLR. By changing to RSA with MPR, they will gain the following advantages:

- More accurate models

- Reduced bias

- Ability to describe complex behaviors such as interactions and other kinds of nonlinearity. 
ANNs are a powerful and increasingly popular modeling technique that is often described as a form of "artificial intelligence." It can be thought of as having layers of functions as complex as MPR models between the multiple inputs (independent variables) and the output (the dependent variable).

ANNs typically have complex structures with a very large number of adjustable coefficients. This makes them susceptible to overfitting (also known as "memorizing the noise"). It also makes an ANN model difficult to communicate to others. You can't write it down as a closed-form equation, like you can with a multivariate polynomial. It is common for researchers who produce ANN models to describe how well it worked. But they don't print the form of the model for others to use. Because the ANN model cannot be easily expressed in mathematical form, it is difficult to perform standard kinds of analysis, such as computing derivatives for sensitivity analysis. You cannot provide the model to someone so they could compute it in the cell of a spreadsheet. Essentially, ANN models can provide predictions, but little other information about the behavior of the system being modeled. For these reasons, ANNs may be called "the blackest of the black box models" (Vaccari, 2021) [32].

\subsection{Neural Network (NN)}

The Neural platform implements a fully connected multi-layer perceptron with one or two layers. Use neural networks to predict one or more response variables using a flexible function of the input variables. Neural networks can be very good predictors when it is not necessary to describe the functional form of the response surface, or to describe the relationship between the inputs and the response.

The Neural Network model is applied between this data on two types of dryland classes (sparse and degraded areas) from 2001 to 2013. This approch is used to predict future scenario from February by algorithm. The Neural network is used to predict one or more response variables using a flexible function of the input variables. Neural networks can be very good predictors when it is not necessary to describe the functional form of the response surface, or to describe the relationship between the inputs and the response (Proust, 2016) [33].

A model report is created for every neural network model. Measures of fit appear for the training and validation sets. Additionally, confusion statistics appear when the response is nominal or ordinal.

Generalized Rsquare: A measure that can be applied to general regression models. It is based on the likelihood function $\mathrm{L}$ and is scaled to have a maximum value of 1 . The value is 1 for a perfect model and 0 for a model no better than a constant model. The Generalized RSquare measure simplifies to the traditional RSquare for continuous normal responses in the standard least squares setting.

Entropy Rsquare: Compares the log-likelihoods from the fitted model and the constant probability model appears only when the response is nominal or ordinal.

Rsquare: Gives the RSquare for the model.

RMSE: Gives the root mean square error. When the response is nominal or ordinal, the differences are between 1 and $\mathrm{p}$ (the fitted probability for the response level that actually occurred).

Mean Abs Dev: The average of the absolute values of the differences between the response and the predicted response. When the response is nominal or ordinal, the differences are between 1 and $p$ (the fitted probability for the response level that actually occurred).

Misclassification Rate: The rate for which the response category with the highest fitted probability is not the observed category. Appears only when the response is nominal or ordinal.

-LogLikelihood: Gives the negative of the loglikelihood. See the Fitting Linear Models book.

SSE: Gives the error sums of squares. Available only when the response is continuous.

Sum Freq: Gives the number of observations that are used. If you specified a Freq variable in the Neural launch window, Sum Freq gives the sum of the frequency column. If there are multiple responses, fit statistics are given for each response, and an overall Generalized RSquare and negative Log-Likelihood is given. 


\section{Results}

\subsection{Performance of Polynomial Regression (MPR) test in ecological modeling}

This research used a quantitative approach. First hypothesis testing in this research was carried out using the Polynomial Regression (MPR) analysis technique with the help of the Smart TaylorFit program.

The relationships established between cumulative rainfall from September to February and NDVI averages from February to April under this prototype is a polynomial regression (Fig. $3 \& 4$ ). These data are highly significant for the period between 2001 and 2013. During the following years the NDVI values dropped. This index easily saturates and the polynomial model poorly estimates plant biomass when the vegetation index is stable and below 0.2 .

The results obtained show that the polynomial regressions between averages NDVI and cumulative precipitation of sparse and degraded lands are highly significant $(\mathrm{Rsq}=0.76 /$ Max Err $=100.49 /$ RMSE $=38.703$ ).

\section{Matlab function:}

Rainfall $=(\mathbf{1 3 9 1 . 5 9 2 8 0 4 7 5 0 1 8 1} *$ NDVI $)+(-$ 72.99907999908811)

Éq 4.

The same results show the perfect relationship between rainfall and NDVI, but the error is less than first regression $(\mathrm{Rsq}=0.76 / \mathrm{Max}$ Err $=0.056 /$ RMSE $=0.024)$.

\section{Matlab function:}

$\mathrm{NDVI}=(\mathbf{0 . 0 0 0 5 4 5 2 5 7 2 8 4 1 5 5 0 7 8 8} *$ rainfall $(\mathrm{mm}))+(\mathbf{0 . 0 8 6 0 3 2 4 9 6 4 6 6 6 5 2 5 4})$

Éq 5.

Based on the estimation results of the MPR model in the pictures above, it can be seen that all indicators in each regression have a loading simple code to estimate each index. Other recent results are very promising for an estimation of NDVI from station rainfall. Polynomial regressions are perfectly established between NDVI and station-derived rainfall with $\left(\mathrm{R}^{2}=0.73\right)$ (Zbiri et al., 2019b) [34].

\section{TaulorFit Response Surface Analysis - with stepwise Multivariate Polynomial Regression}

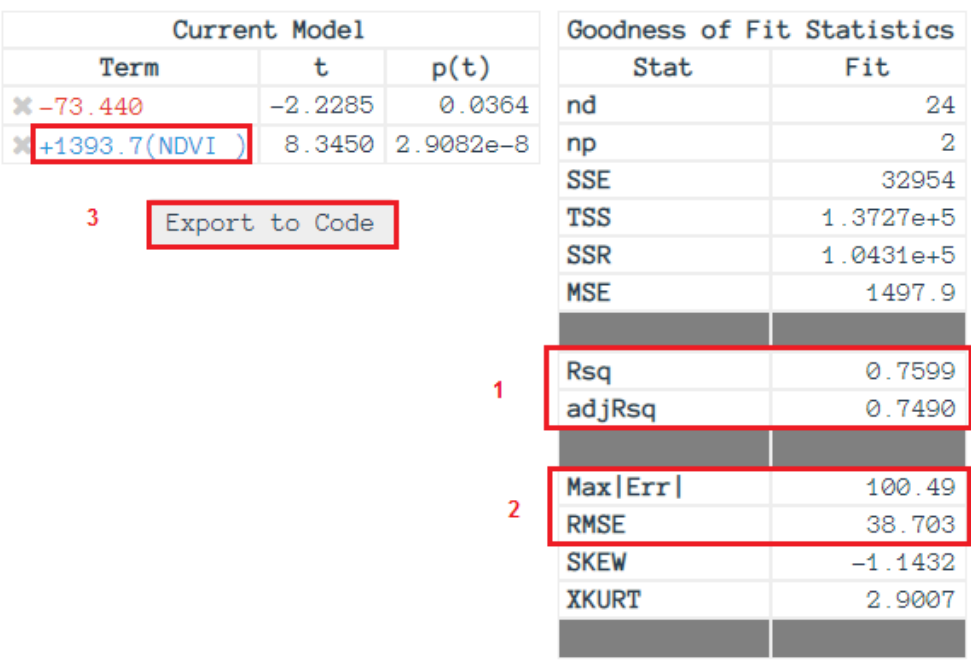

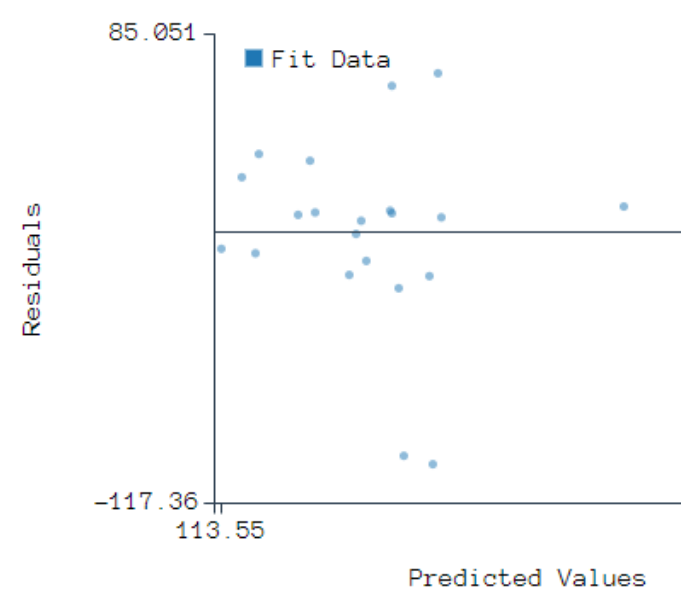

Fig. 3 Test 1: Polynomial regression using eMODIS NDVI (February to April) and ECMWF rainfall (September to February) with (24 Fit 2001 to 2013) via TaylorFit AI. 


\section{TaylorFit Response Surface Analysis - with stepwise Shultivariate Polynomial Regression}

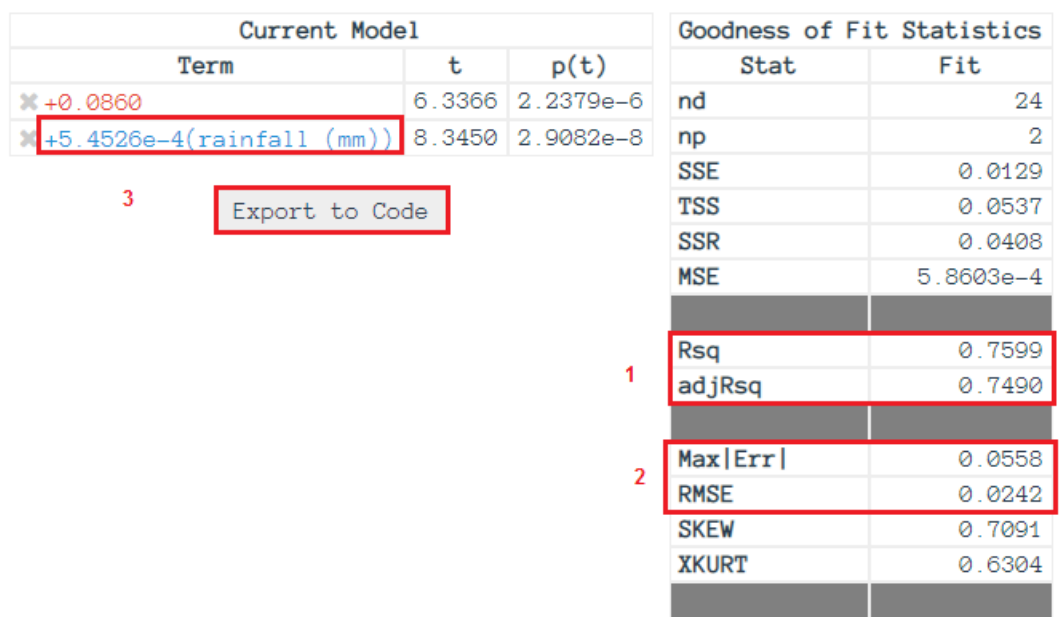

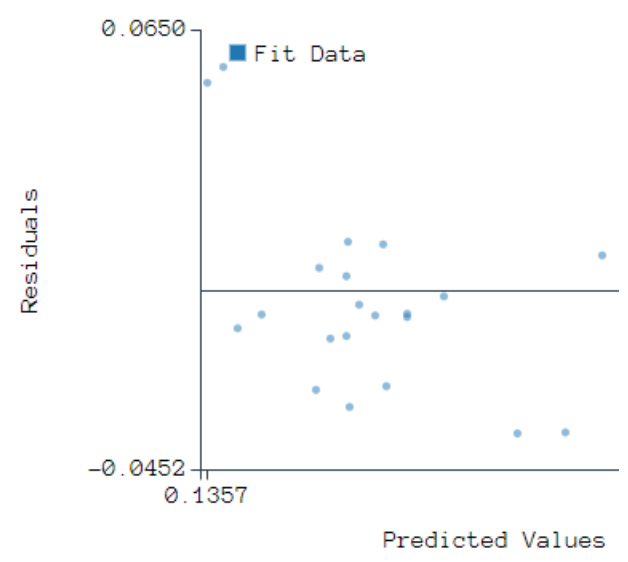

Fig. 4 Test 1: Polynomial regression using ECMWF rainfall (September to February) and eMODIS NDVI (February to April) with (24 Fit 2001 to 2013) via TaylorFit AI.

\subsection{Performance of Neural Network (NN) test in ecological modeling}

At the Neural Network test, the training and the validition steps was carried out (Fig. 5 \& 6). Based on the results of the validation model, $\mathrm{R}$ coefficient and RMSE show a good regression between the NDVI and rainfall data $(\mathrm{Rsq}=0.71 / \mathrm{RMSE}=0.025)$ and $(\mathrm{Rsq}=0.98 / \mathrm{RMSE}=10.52)$.

The hypothesis testing to estimate rainfall from NDVI is stronger than inverted pattern. The SAS final layer code at the inner model phase is carried out based on the significant value $\mathrm{H} 1, \mathrm{H} 2, \mathrm{H} 3$ and THETA1 code. The formulas for a simple experiment are very complex and the interpretation is long.

The SAS predictive and specialized model profiler indicates NDVI values ranging from 0.15 to 0.3 and cumulative rainfall from 50 to $400 \mathrm{~mm}$. For all consecutive $174-193 \mathrm{~mm}$ rainfall bins, indicate 0.18-0.19 mean NDVI.

Oludare et al. 2018 study covers many applications of ANN techniques in various disciplines which include computing, science, engineering, medicine, environmental, agriculture, mining, technology, climate, business, arts, and nanotechnology, etc. The study assesses ANN contributions, compare performances and critiques methods. The study found that neural-network models such as feedforward and feedback propagation artificial neural networks are performing better in its application to human problems. Therefore, we proposed feedforward and feedback propagation ANN models for research focus based on data analysis factors like accuracy, processing speed, latency, fault tolerance, volume, scalability, convergence, and performance. Moreover, we recommend that instead of applying a single method, future research can focus on combining ANN models into one network-wide application.

This is a survey of neural network applications in the real-world scenario. It provides a taxonomy of artificial neural networks (ANNs) and furnish the reader with knowledge of current and emerging trends in ANN applications research and area of focus for researchers. Additionally, the study presents ANN application challenges, contributions, compare performances and critiques methods. The study covers many applications of ANN techniques in various disciplines which include computing, science, engineering, medicine, environmental, agriculture, mining, technology, climate, business, arts, and nanotechnology, etc [35].

A multitude of applications of Neural Network proves its power in artificial language. Thus, in the modeling of disasters can use this type of program to managing a risk in time and space. 
In an arid environment and to predict climatic or natural senario, the algorithm of SAS NN created in this study is very reliable for several applications see the disaggregation of a product of ECMWF in $250 \mathrm{~m}$ (resolution of NDVI). As we have used this data from the European center and from emodis, we can use any other data.
The NN results show a margin equal to $98 \%$, which explains why our method is important for estimating rainfall from NDVI data.

In the climatic context, the water stress related to the lack of rainfall for all Moroccan drylands can be monitored artificially.

These interfaces to allow them to provide ecosystem services that meet human demands for social development.

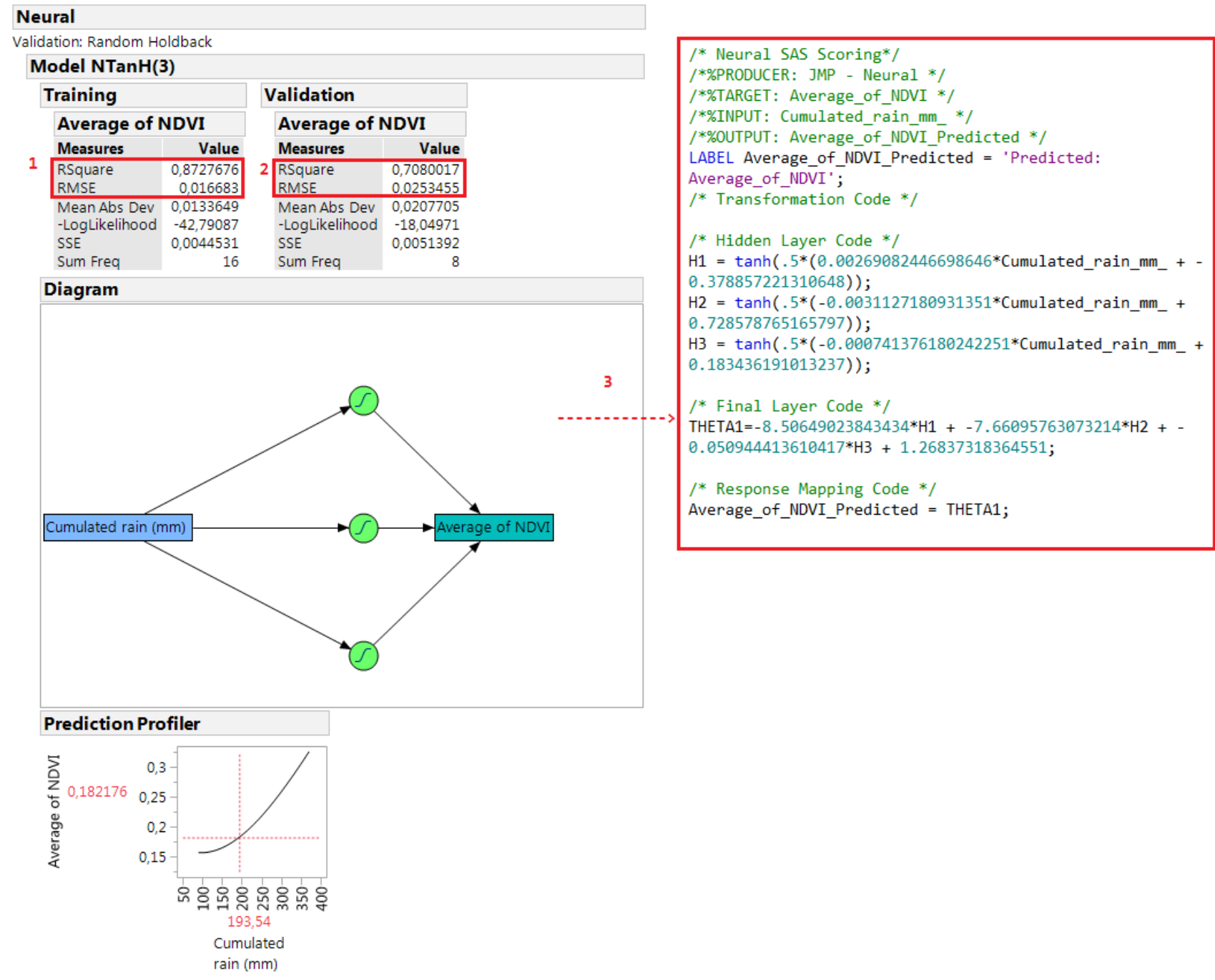

Fig. 5 Test 2: Neural Network model using ECMWF rainfall (September to February) and eMODIS NDVI (February to April) with (24 Fit 2001 to 2013) via SAS AI. 


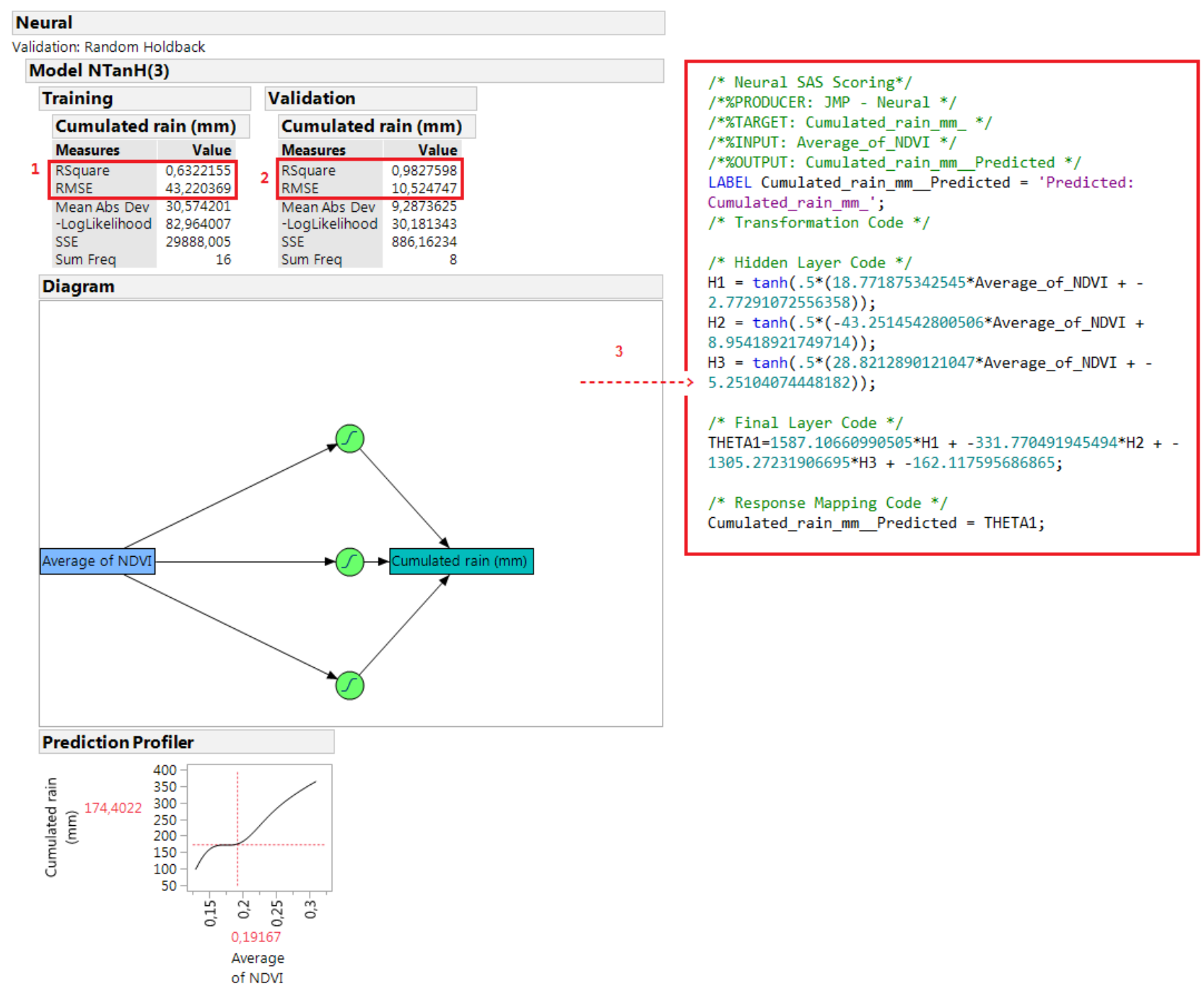

Fig. 6 Test 2: Neural Network model using eMODIS NDVI (February to April) and ECMWF rainfall (September to February) with (24 Fit 2001 to 2013) via SAS AI.

\section{Discussion}

From the simple data and two statistical hypotheses it was possible to obtain valuable information about the intelligent analysis systems. These programs based on precision algorithms prove their power in the projection of future scenarios in different fields. Raising a problem and studying the hypothesis to solve it now is easy with virtual work. why each product is always provided with a validation report.

Thus, the authors must be careful to use these data taking into account the recommendations of the experts who have executed the algorithm of the product (Rousson, 2013; Lejeune, 2010; Weiers \& Heinz, 2011; Good \& Hardin, 2012) [36, 37, 38, 39].
Currently with the pandemic and after the remote work are the future research, teaching and entrepreneurship. According to researchers, before leaping to the conclusion that (Virtual Work; VW) saves leasing expenses and delights digital workers. This is vital that the research community take part to solve of environmental risk can have economic and social.

Furthermore, government must build solid conceptual frameworks human capital for what leads to detracts from virtual work efficiency, which is focused on the essence of work done by workers of multiple grades, in order to formulate a new approach of managing productivity. 


\section{Conclusions and perspectives}

Overall, both Artificial Intelligence AIs (TaylorFit or SAS Neural Network) have almost the same data processing. The first one requires the use of a CSV, the second one use Excel file with conversion of commas numbers into points.

Based on the results obtained in this research, the performance of the polynomial model proves that the regressions between NDVI and precipitation remain highly significant as evidenced by $\mathrm{R}^{2}=0.76$. Also, it appears that thes algorithms of matlab function via TaylorFit are simple and easy to use.

For example, the adoption of these models should help decision-makers to alert livestock farmers to the risk of rangeland drought and to forecast forage import requirements in advance.

The results clearly confirm that the NDVI can be used as an indicator of the quality of rainfall estimates at the continental/ regional scale and allow a rapid detection of major over- and underestimations of the two rainfall datasets examined for the African continent (Rjas et al., 2011) [25].

The following the next approach of SAS Neural Network it is could be involve in the disaggregation of ECMWF rainfall data with NDVI at $250 \mathrm{~m}$ resolution.

\section{Acknowledgments: The authors gratefully acknowledge support from the WSEAS Editor in Chef. We also thank Dominique Haesen and Prof. DAVID A.VACCARI and two anonymous reviewers for their useful suggestions that significantly improved the readability of the paper.}

\section{References:}

[1]. Hofste, R.W., Reig, P. \& Schleife, L. 2019. 17 Countries, Home to One-Quarter of the World's Population, Face Extremely High Water Stress. World Water Resources Institute.

[2]. Gommes, R., El Hairech, T., Rosillon, D., Balaghi, R, 2009. Impact of climate change on agricultural yields in Morocco. World Bank - Morocco study on the impact of climate change on the agricultural sector. Food and Agriculture Organization of the United Nations (FAO). Roma, Italy. 105p.

[3]. Dutta, D., Kundu, A., Patel, N.R., Saha, S.K. \& Siddiqui, A.R, 2015. Assessment of agricultural drought in Rajasthan (India) using remote sensing derived vegetation condition index (VCI) and Standardized Precipitation Index (SPI). The Egyptian Journal of Remote Sensing and Space Sciences, 18, 53-63.

[4]. Hachmi, A., Andich, K., El Alaoui-Faris, F.E., Mahyou, H, 2018. Improvement of the state of vegetation and soil fertility of arid rangelands in Morocco by restoration and rehabilitation techniques. Revue d'Écologie, $13 \mathrm{p}$.

[5]. Rojas-Downing, M.M., Nejadhashemi, A.P., Harrigan, T., Woznicki, S.A, 2017. Climate change and livestock: Impacts, adaptation, and mitigation. Climate Risk Management, 19p.

[6]. Berkat, O. \& Tazi M, 2004, Profil fourrager Maroc, FAO, 29p.

[7]. Zbiri, A., Haesen, D., El Alaoui-faris, F.E., Hachmi, A. \& Vaccari, D.A, 2021. Algorithm theoretical for FAPAR and DMP calibration using remote sensing and field data in Moroccan arid areas. International Journal of Environmental Science. Vol 6, $12 \mathrm{pp}$.

[8]. Xu, P., Zhou, T., Zhao, X., Luo, H., Gao, S., Zheng, Li., Leyao, C, 2018. Diverse responses of different structured forest to drought in Southwest China through remotely sensed data. MDPI, Int. J. Appl. Earth Obs. Geoinf, 16p.

[9]. Coppin, P., Jonckheere, I., Nackaerts, K., Muys, B., Lambin, E, 2004. Review ArticleDigital Change Detection Methods in Ecosystem Monitoring: A Review. Int. J. Remote Sens., 25, 1565-1596.

[10]. Hecheltjen, A., Thonfeld, F., Menz, $\mathrm{G}, 2014$. Recent Advances in Remote Sensing Change Detection-A Review. In Land Use and Land Cover Mapping in Europe; Manakos, I., Braun, M., Eds.; Remote Sensing and Digital Image Processing; Springer: Dordrecht, The Netherlands, Volume 18, pp. 145-178. ISBN 978-94-007-7968-6.

[11]. Gorelick, N., Hancher, M., Dixon, M., Ilyushchenko, S., Thau, D., Moore, R, 2017. Google Earth Engine: PlanetaryScale Geospatial Analysis for Everyone. Remote Sens. Environ., 202, 18-27.

[12]. Jackson, K.R., Ramakrishnan, L., Muriki, K., Canon, S., Cholia, S., Shalf, J., 
Wasserman, H.J., Wright, N.J, 2010. Performance Analysis of High Performance Computing Applications on the Amazon Web Services Cloud. In Proceedings of the 2010 IEEE Second International Conference on Cloud Computing Technology and Science, Indianapolis, IN, USA, pp. 159168.

[13]. Nemani, R, 2012. NASA Earth Exchange: Next Generation Earth Science Collaborative. Int. Arch. Photogramm. Remote Sens. Spat. Inf. Sci, XXXVIII8/W20, 17.

[14]. Tamiminia, H., Salehi, B., Mahdianpari, M., Quackenbush, L., Adeli, S., Brisco, B, 2020. Google Earth Engine for Geo-Big Data Applications: A MetaAnalysis and Systematic Review. ISPRS J. Photogramm. Remote Sens. 164, 152-170.

Rouse, J.W., Haas, R.H., Schell, J.A., Deering, D.W, 1974. Monitoring vegetation systems in the Great Plains with ERTS. NASA Technical Reports Server, (1) : Sect. A, 309-317.

[16]. Guo, X., 2002. Discrimination of Saskatchewan Prairie Ecoregions Using Multitemporal 10 day Composite NDVI data. Prairie Perspectives, vol 5, p.p. 174186.

[17]. Zbiri, A., Haesen, D., El Alaouifaris, F.E. \& Mahyou, H, 2019a. Drought monitoring using soil water index and normalized difference vegetation index time series in Moroccan rangelands. WSEAS TRANSACTIONS on ENVIRONMENT and DEVELOPMENT. Vol 15, \#30, pp. 261-278.

[18]. Nicholson, S.E. \& Farrar, T.J, 1994. The influence of soil type on the relationships between NDVI, rainfall, and soil moisture in semiarid Botswana. I. NDVI response to rainfall. Remote Sens. Environ, 50(2), 107-120.

[19]. Farrar, T.J., Nicholson, S.E., Lare, A.R, 1994. The influence of soil type on the relationships between NDVI, rainfall and soil moisture in semiarid Botswana. II. NDVI response to soil moisture. Remote Sens of Environ, 50, 121-133.

[20]. Thenkabail, P.S., Gamage, M.S.D.N. \& Smakhtin, V.U, 2004. The Use of Remote-Sensing Data for Drought
Assessment and Monitoring in Southwest Asia.

[21]. Arshad, S., Morid, S., Mobasheri, M.R. \& Agha Alikhani, M, 2008. Development of Agricultural Drought Risk Assessment Model for Kermanshah Province (Iran), using satellite data and intelligence methods. Option Mediterrianeennes, Series A, No: 80.

[22]. Hasan, M. \& Saiful Islam, A.K.M, 2011. Drought Assessment Using Remote Sensing and Gis in North-West Region of Bangladesh. 3rd International Conference on Water \& Flood Management (ICWFM2011).

[23]. Brian, D., Martha, W., Anderson, C. \& James, P, 2012. Remote Sensing of Drought. Taylor \& Francis Group.

[24]. Martiny, N., Camberlin, P., Richard, Y. \& Philippon, N, 2006. Compared regimes of NDVI and rainfall in semi-arid regions of Africa. International Journal of Remote Sensing, Vol. 27, No. 23, 5201-5223.

[25]. Rojas, O., Rembold, F., Delince, J. \& Leo O, 2011. Using the NDVI as auxiliary data for rapid quality assessment of rainfall estimates in Africa. International Journal of Remote Sensing, Vol. 32, No. 12, 3249-3265.

[26]. Woods, A, 2006. Medium-Range Weather Prediction-The European Approach: The Story of the CEPMMT. Springer: New York, NY; 270 pp.

[27]. Rojas, O., Rembold, F., Royer, A. \& Negre, T, 2005. Real-time agro meteorological crop yield monitoring in Eastern Africa. Agronomy for Sustainable Development, 25, pp. 63-77.

[28]. Rojas, O, 2007. Operational maize yield model development and validation based on remote sensing and agrometeorological data in Kenya. International Journal of Remote Sensing, 28, pp. 37753793.

[29]. ECMWF https://www.ecmwf.int/.

[30]. Jenkerson, C., Maiersperger, T. \& Schmidt, G., 2010. eMODIS. A UserFriendly Data Source.

[31]. Mayaux, P., Bartholome, E., Fritz, S. \& Belward, A, 2004. A new land-cover map of Africa for the year 2000. 
[32]. Vaccari, D.A, 2021. TaylorFit Users' Manual, http://www.taylorfitrsa.com/.

[33]. Proust, M, 2016. JMP $P^{\circledR} \quad 13$ Predictive and Specialized Modeling. JMP, A Business Unit of SAS, SAS Campus Drive, Cary, NC 27513. USA.

[34]. Zbiri, A., Hachmi, A., Haesen, D., El Alaoui-faris, F.E. \& Mahyou H, 2019 b. Efficiency of climate and remote sensing data to drought monitoring in arid areas: Case of Eastern Morocco. WSEAS TRANSACTIONS on ENVIRONMENT and DEVELOPMENT. Vol 15, \#42, pp. 378-394.

[35]. Oludare, I.A., Aman, J., Abiodun, E.O., Kemi, V.D., Nachaat, A.M., Humaira, A, 2018. State-of-the-art in artificial neural network applications: A survey, Heliyon, Volume 4, Issue 11, e00938.

[36]. Rousson, V, 2013. Statistics applied to life sciences. Paris, Berlin, Heidelberg, New York, Hong Kong, Londres, Milan, Tokyo, Springer, 327p.

[37]. Lejeune, M, 2010. Statistics: Theory and applications. 2e éd, Paris, Berlin, Heidelberg. New York, Hong Kong, Londres, Milan, Tokyo, Springer, 448.

[38]. Weiers, R.M. \& Heinz, H.J., 2011. Introduction to Business Statistics, USA, Joe Sabatin, 892p.

[39]. Good, P. I. \& Hardin, J.W., 2012. Common Errors in Statistics and How to Avoid Them. 4 e éd, Hoboken, New Jersey, John Wiley et Sons, 352p.

\section{Creative Commons Attribution License 4.0 (Attribution 4.0 International, CC BY 4.0)}

This article is published under the terms of the Creative Commons Attribution License 4.0 https://creativecommons.org/licenses/by/4.0/deed.en_US 